\title{
ELEMENTARY PROOFS OF PEANO'S EXISTENCE THEOREM
}

\author{
M. A. DOW and R. VÝBORNÝ
}

(Received 6 March 1972)

Communicated by J. Virsik

\begin{abstract}
An "elementary" proof of Peano's existence theorem is given that, in addition to avoiding the Ascoli lemma, relies neither on Dini's theorem, nor on uniform continuity of the right hand side of $\phi^{\prime}=\mathrm{f}(t, \phi)$. It is based on superfunctions. Also, another standard proof of that theorem, based on approximation of the right hand side, is made elementary.
\end{abstract}

\section{Introduction}

Recently, the question of an elementary proof of Peano's existence theorem has been discussed. (See Kennedy [1] and Walter [4].) Peano's theorem states that the initial value problem

$$
\phi^{\prime}=f(t, \phi), \phi\left(t_{0}\right)=x_{0},
$$

has a solution given only that $f$ is continuous. (See Peano [2] and also Perron [3].) In [4], Walter gave his own constructive proof using a monotone sequence of approximate solutions, thus avoiding equicontinuous families and the Ascoli lemma. Grunsky [5] has also given a constructuve proof. There is no doubt that Walter's proof is "elementary". However, in this paper, we show that not only Ascoli's lemma can be avoided but also any reference to uniform continuity of $f$ and to theorems on uniform convergence. Theorems $\mathbf{A}$ and $\mathbf{B}$ given in this paper guarantee the existence of the maximal solution to the initial value problem (1) under the type of hypothesis used by Perron in [3]. The proof of Theorem A uses a general class of superfunctions in the spirit of Perron's original proof, but can be described as "elementary".

It is well known that a proof of the existence theorem for the initial value problem (1) can be based on approximation of $f$ by functions $f_{n}$ for which the initial value problem $\phi^{\prime}=f_{n}(t, \phi), \phi\left(t_{0}\right)=\phi_{0}$ has a solution. It will be apparent from the proof of Theorem B that Ascoli's lemma can be avoided in this procedure as well. In fact, the whole proof can be made "elementary". 


\section{Statement of the problem}

THEOREM A. Let $w_{1}$ and $w_{2}$ be continuous functions on an interval $J=\left[t_{0}, T\right]$ such that the right hand derivatives $D_{+} w_{1}$ and $D_{+} w_{2}$ exist on $\left[t_{0}, T\right), w_{1}\left(t_{0}\right)=w_{2}\left(t_{0}\right)=x_{0}$, and $w_{1}(t) \leqq w_{2}(t)$ on $J$. Let $f$ be defined and continuous on the domain $D=\left\{(x, t): t_{0} \leqq t \leqq T, w_{1}(t) \leqq x \leqq w_{2}(t)\right\}$. Suppose that $D_{+} w_{1}(t) \leqq f\left(t, w_{1}(t)\right)$ and $D_{+} w_{2}(t) \geqq f\left(t, w_{2}(t)\right)$ on $J$. Then there exists a maximal solution $\psi$ to the problem $\phi^{\prime}=f(t, \phi), \phi\left(t_{0}\right)=x_{0}$ on $J$, and $w_{1}(t) \leqq \psi(t) \leqq w_{2}(t)$ on $J$

Without loss of generality, we set $t_{0}=x_{0}=0$ and $T>0$ in the rest of the paper.

In the proofs, a crucial role is played by a theorem discussed by Walter in remark (c) of [4]. For a complete discussion of theorems of this type see Walter [6], chapter 2. For convenience, we state what we need as a lemma.

LeMma. Let $f$ and $g$ be continuous functions on $[a, b]$ that are differentiable from the right and satisfy $f(a) \geqq g(a)$. If $D_{+} f\left(t_{0}\right)>D_{+} g\left(t_{0}\right)$ at each point $t_{0}$ of $[a, b]$ at which $f\left(t_{0}\right)=g\left(t_{0}\right)$, then $f \geqq g$ on $[a, b]$.

In order to facilitate the proofs, we expand the domain of $f$ to $J \times R$ by defining

$$
f(t, x)= \begin{cases}f\left(t, w_{2}(t)\right) & \text { if } x>w_{2}(t) \\ f\left(t, w_{1}(t)\right) & \text { if } x<w_{1}(t) .\end{cases}
$$

Let $|f(t, x)| \leqq M$ on $J \times R$.

\section{Proof of Theorem A}

Let $\Omega$ be the set of all continuous functions $\phi$ on $J$ that are differentiable from the right on $[0, T)$ and satisfy $\phi(0)=0$ and $D_{+} \phi(t)>f(t, \phi(t))$ on $[0, T)$. We call a member of $\Omega$ a superfunction. Since $w_{2}(t)+\varepsilon t$ is a superfunction for each $\varepsilon>0, \Omega \neq \varnothing$. Since the lemma implies that $\phi(t) \geqq w_{1}(t)$ on $J, w_{1}$ is a lower bound for $\Omega$. Define the function $\psi$ on $J$ by $\psi(t)=\inf \{\phi(t): \phi \in \Omega\}$. It follows that $w_{1} \leqq \psi \leqq w_{2}$.

To show that $\psi$ is Lipschitz continuous with constant $M$, one can follow Perron's proof (see [3], pp. 474-475) or slightly simplify it by using the lemma of $\S 1$ instead of the theorem in the footnote on page 474 in [3].

We show now that $\psi$ is a solution. It is sufficient to prove that if $t_{1}, t_{2} \in J$ and $t_{1}<t_{2}$, then

$$
\psi\left(t_{2}\right)-\psi\left(t_{1}\right)=\int_{t_{1}}^{t_{2}} f(t, \psi(t)) d t
$$

Clearly, $\psi(0)=0$. Fix $t_{1}$ and $t_{2}$. Define 


$$
F(t)=\psi(t)-\psi\left(t_{1}\right)-\int_{t_{1}}^{t} f(s, \psi(s)) d s,
$$

where $t_{1} \leqq t \leqq t_{2}$. Equality (2) is equivalent to the equality

$$
L \equiv \frac{F\left(t_{2}\right)-F\left(t_{1}\right)}{t_{2}-t_{1}}=0 .
$$

We show that the assumption $L \neq 0$ leads to a contradiction. By induction, we define a sequence of subintervals $\left[a_{k}, b_{k}\right]$ of $\left[t_{1}, t_{2}\right]$ as follows. Let $a_{1}=t_{1}, b_{1}=t_{2}$. Given $t_{1} \leqq a_{k-1}<b_{k-1} \leqq t_{2}$, there exist $a_{k}$ and $b_{k}$ with $a_{k-1}<a_{k}<b_{k}<b_{k-1}$, $b_{k}-a_{k} \leqq \frac{1}{3}\left(b_{k-1}-a_{k-1}\right)$ and

$$
\frac{F\left(b_{k}\right)-F\left(a_{k}\right)}{b_{k}-a_{k}}=L
$$

(see, for example, Aziz and Diaz [7]); that is,

$$
\phi_{k} \equiv \frac{\psi\left(b_{k}\right)-\psi\left(a_{k}\right)}{b_{k}-a_{k}}=\frac{1}{b_{k}-a_{k}} \int_{a_{k}}^{b_{k}} f(s, \psi(s)) d s+L .
$$

Let $\bar{t}$ be the unique point common to all intervals $\left[a_{k}, b_{k}\right]$. Continuity of $f$ at $(\bar{t}, \psi(\bar{t}))$ implies the existence of an open, spherical neighborhood $N$ of $(\bar{t}, \psi(\bar{t}))$ with $|f(t, x)-f(\bar{t}, \psi(\bar{t}))|<\frac{1}{2}|L|$ for $(t, x)$ in $N$. Then, there is $k_{0}$ so large that $(t, \psi(t)) \in N$ if $a_{k_{0}} \leqq t \leqq b_{k_{0}}$ and, also, that for all $k>k_{0}$, the graph of $l_{k}(t)$ $=\left[f(\bar{t}, \psi(\bar{t}))+\frac{1}{2} L\right]\left(t-a_{k}\right)+\psi\left(a_{k}\right)$ lies in $N$ for $a_{k} \leqq t \leqq b_{k}$. In fact, for $a_{k} \leqq t$ $\leqq b_{k},|\bar{t}-t| \rightarrow 0$ as $k \rightarrow \infty$ and

$$
\left|\psi(\bar{t})-l_{k}(t)\right| \leqq\left|\psi(\bar{t})-\psi\left(a_{k}\right)\right|+\left(b_{k}-a_{k}\right)\left|f(\bar{t}, \psi(\bar{t}))+\frac{1}{2} L\right| \rightarrow 0
$$

as $k \rightarrow \infty$.

Let $k>k_{0}$ be fixed. We distinguish between two cases: $(\alpha) L<0$ and $(\beta)$ $L>0$.

( $\alpha$ ) Suppose $L<0$. Then

$$
\phi_{k}=\frac{1}{b_{k}-a_{k}} \int_{a_{k}}^{b_{k}} f(s, \psi(s)) d s-|L|=f\left(t^{\prime}, \psi\left(t^{\prime}\right)\right)-|L|
$$

for some $t^{\prime} \in\left(a_{k}, b_{k}\right)$. Since $\left(t^{\prime}, \psi\left(t^{\prime}\right)\right) \in N$, we have $\phi_{k}<f(\bar{t}, \psi(\bar{t}))-\frac{1}{2}|L|$. This means that $\psi\left(b_{k}\right)<l_{k}\left(b_{k}\right)$. Therefore, there exists $\phi \in \Omega$ such that $\phi\left(b_{k}\right)<l_{k}\left(b_{k}\right)$. There are two possibilities. Either $\phi(t)<l_{k}(t)$ for all $t \in\left[a_{k}, b_{k}\right]$, or $\phi\left(t_{0}\right)=l_{k}\left(t_{0}\right)$ for some $t_{0} \in\left[a_{k}, b_{k}\right)$. In the first case, $\phi\left(a_{k}\right)<l_{k}\left(a_{k}\right)=\psi\left(a_{k}\right)$, contradicting the definition of $\psi$. In the second case, we can apply the lemma of $\$ 1$ on the interval $\left[t_{0}, b_{k}\right]$ with $f=\phi$ and $g=l_{k}$, since $D_{+} \phi>f(t, \phi)>f(\bar{t}, \psi(\bar{t}))-\frac{1}{2}|L|=l_{k}^{\prime}$. Thus $l_{k} \leqq \phi$ on $\left[t_{0}, b_{k}\right]$, in particular, $l_{k}\left(b_{k}\right) \leqq \phi\left(b_{k}\right)$, contradicting our choice of $\phi$. Therefore, $L<0$ is impossible. 

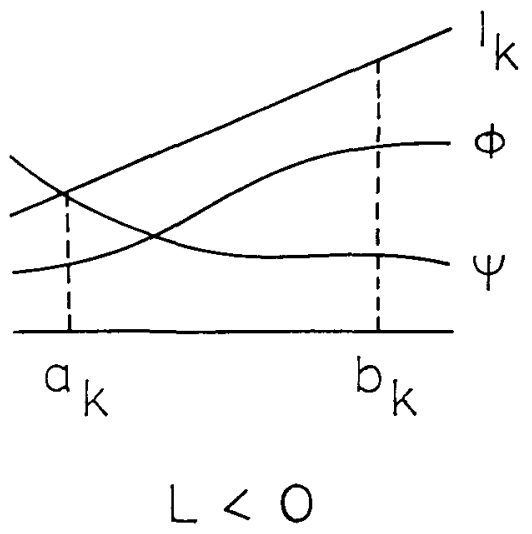
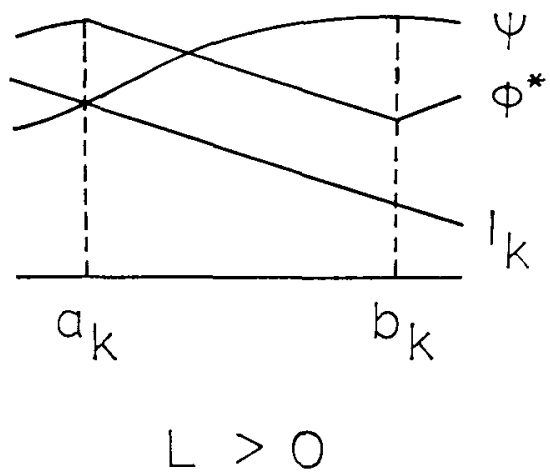

$(\beta)$ Suppose $L>0$. Here, $\phi_{k}>f(\bar{t}, \psi(\bar{t}))+\frac{1}{2} L$. Thus, $\psi\left(b_{k}\right)>l_{k}\left(b_{k}\right)$. There is $\phi \in \Omega$ such that $\left(a_{k}, \phi\left(a_{k}\right)\right) \in N$ and $\phi\left(a_{k}\right)-\psi\left(a_{k}\right)<\psi\left(b_{k}\right)-l_{k}\left(b_{k}\right)$. Then the line $l_{k}^{*}(t)=\left[f(\bar{t}, \psi(\bar{t}))+\frac{1}{2} L\right]\left(t-a_{k}\right)+\phi\left(a_{k}\right)$ satisfies $l_{k}^{*}\left(b_{k}\right)<\psi\left(b_{k}\right)$. Define $\phi^{*}$ as follows:

$$
\phi^{*}(t)= \begin{cases}\phi(t) & \text { if } 0 \leqq t \leqq a_{k}, \\ l_{k}^{*}(t) & \text { if } a_{k}<t \leqq b_{k}, \\ (M+1)\left(t-b_{k}\right)+\phi^{*}\left(b_{k}\right) & \text { if } b_{k}<t \leqq T .\end{cases}
$$

Then $\phi^{*} \in \Omega$, but $\phi^{*}\left(b_{k}\right)<\psi\left(b_{k}\right)$, a contradiction.

This completes the proof that $\psi$ is a solution. That $\psi$ is maximal is an easy consequence of the lemma.

\section{Remarks}

REMARK 1. If, in the hypothesis, we replace the words " $D_{+} w_{1}$ and $D_{+} w_{2}$ exist on $\left[t_{0}, T\right)$ ' by " $D_{-} w_{1}$ and $D_{-} w_{2}$ exist on $\left(t_{0}, T\right]$ ', and the words ' $D_{+} w_{1}(t)$ $\leqq f\left(t, w_{1}(t)\right)$ and $D_{+} w_{2}(t) \geqq f\left(t, w_{2}(t)\right)$ " by " $D_{-} w_{1}(t) \leqq f\left(t, w_{1}(t)\right)$ and $D_{-} w_{2}(t)$ $\geqq f\left(t, w_{2}(t)\right)$ ", then we may modify the proof to deduce the existence of a solution $\psi$ to the problem $\phi^{\prime}=f(t, \phi), \phi\left(t_{0}\right)=x_{0}$ on $J$ and $w_{1}(t) \leqq \psi(t) \leqq w_{2}(t)$ on $J$. In the proof, this is done by letting $\Omega$ be the set of continuous functions on $J$ that are differentiable from the left and satisfy $\phi(0)=0$ and $D_{-} \phi(t)<f(t, \phi(t))$ on $(0, T]$. We let $\psi(t)=\sup \{\phi(t): \phi \in \Omega\}$. The main feature distinguishing this proof from that for superfunctions is that a lemma analogous to that of $\S 1$ is weaker (see Walter [6]). In order to conclude that $f \geqq g$ on an interval $[a, b]$ one must assume $f(a)>g(a)$. Thus the proof must be modified whenever the lemma is applied. Note that this weaker lemma is not sufficient to show that $\psi$ is minimal.

REMARK 2 . In proving that $\psi$ is a solution, one may use a mean value theorem for continuous functions by Diaz and Výborný (see [8], top of p. 115) instead of the method of nested intervals. 


\section{A second proof}

THEOREM B. Let the hypothesis of theorem $A$ hold. Suppose for each $n$ there is a function $f_{n}$ defined on $D$ such that $\left|f(t, x)-f_{n}(t, x)\right|<\left(\frac{1}{2}\right)^{n}$ for all $(t, x) \in D$ and the initial value problem

$$
\phi^{\prime}=f_{n}(t, \phi)+\frac{4}{2^{n}}, \phi(0)=0
$$

has a solution $\phi_{n}$ on $J$. Then the sequence $\left\{\phi_{n}\right\}$ converges pointwise to a maximal solution $\psi$ of the initial value problem $\phi^{\prime}=f(t, \phi), \phi(0)=0$, and, moreover, $w_{1} \leqq \psi \leqq w_{2}$.

Proof. Extend the domain $D$ of $f$ and each $f_{n}$ to $J \times R$ as in $\S 1$. Let $F_{n}(t, x)$ $=f_{n}(t, x)+4 / 2^{n}$. It is easy to see that $F_{n}>F_{n+1}$ on $J$. This and the lemma of $\S 1$ imply that $\phi_{n} \geqq \phi_{n+1}$ on $J$. Moreover, $\phi_{n}$ satisfies a Lipschitz condition with constant $M+5 / 2^{n}$. In fact, for $t_{1}$ and $t_{2}$ in $J$,

$$
\begin{aligned}
\left|\phi_{n}\left(t_{2}\right)-\phi_{n}\left(t_{1}\right)\right| & \leqq \int_{t_{1}}^{t_{2}}\left|F_{n}\left(s, \phi_{n}(s)\right)\right| d s \leqq \int_{t_{1}}^{t_{2}}\left(\left|f\left(s, \phi_{n}(s)\right)\right|+\frac{1}{2^{n}}+\frac{4}{2^{n}}\right) d s \\
& \leqq\left(M+\frac{5}{2^{n}}\right)\left|t_{2}-t_{1}\right|
\end{aligned}
$$

where $M$ is an upper bound for $f$. It follows that the sequence $\left\{\phi_{n}\right\}$ is bounded below on $J$. Define $\psi$ on $J$ by $\psi(t)=\inf \phi_{n}(t)$. Then $\psi$ is Lipschitz continuous with constant $M$.

We show that $\psi$ is a solution. Let $\varepsilon>0$ and $t_{1} \in J$. Let $t_{2}$ be any other point of $J$. Then,

$$
\begin{aligned}
\frac{\phi_{n}\left(t_{2}\right)-\phi_{n}\left(t_{1}\right)}{t_{2}-t_{1}} & =\frac{1}{t_{2}-t_{1}} \int_{t_{1}}^{t_{2}} F_{n}\left(t, \phi_{n}(t)\right) d t \\
& =F_{n}\left(t^{\prime}, \phi_{n}\left(t^{\prime}\right)\right)=f_{n}\left(t^{\prime}, \phi_{n}\left(t^{\prime}\right)\right)+\frac{4}{2^{n}}
\end{aligned}
$$

for some $t^{\prime} \in\left(t_{1}, t_{2}\right)$. Now,

$$
\begin{aligned}
\left|f_{n}\left(t^{\prime}, \phi_{n}\left(t^{\prime}\right)\right)-f\left(t_{1}, \psi\left(t_{1}\right)\right)\right| & \leqq\left|f_{n}\left(t^{\prime}, \phi_{n}\left(t^{\prime}\right)\right)-f\left(t^{\prime}, \phi_{n}\left(t^{\prime}\right)\right)\right| \\
& +\left|f\left(t^{\prime}, \phi_{n}\left(t^{\prime}\right)\right)-f\left(t_{1}, \psi\left(t_{1}\right)\right)\right| \equiv A+B .
\end{aligned}
$$

Since $\left|t^{\prime}-t_{1}\right| \leqq\left|t_{2}-t_{1}\right| \rightarrow 0$ as $t_{2} \rightarrow t_{1}$ and

$$
\begin{aligned}
\left|\phi_{n}\left(t^{\prime}\right)-\psi\left(t_{1}\right)\right| & \leqq\left|\phi_{n}\left(t^{\prime}\right)-\phi_{n}\left(t_{1}\right)\right|+\left|\phi_{n}\left(t_{1}\right)-\psi\left(t_{1}\right)\right| \\
& \leqq\left(M+\frac{5}{2^{n}}\right)\left|t^{\prime}-t_{1}\right|+\left|\phi_{n}\left(t_{1}\right)-\psi\left(t_{1}\right)\right| \rightarrow 0
\end{aligned}
$$


as $n \rightarrow \infty$ and $t_{2} \rightarrow t_{1}$, the continuity of $f$ at $\left(t_{1}, \psi\left(t_{1}\right)\right)$ implies the existence of numbers $n_{\varepsilon}$ and $\delta_{\varepsilon}$ such that $B<\varepsilon / 2$ if $\left|t_{2}-t_{1}\right|<\delta_{\varepsilon}$ and $n>n_{\varepsilon}$. In addition, choose $n_{\varepsilon}$ large enough that $1 / 2^{n}<\varepsilon / 2$ for $n>n_{\varepsilon}$, so that $A<1 / 2^{n}<\varepsilon / 2$. Then $A+B<\varepsilon$. Consequently, for all $n>n_{\varepsilon}$ and all $t_{2}$ with $\left|t_{2}-t_{1}\right|<\delta_{\varepsilon}$, we have

$$
f\left(t_{1}, \psi\left(t_{1}\right)\right)+\frac{4}{2^{n}}-\varepsilon<\frac{\phi_{n}\left(t_{2}\right)-\phi_{n}\left(t_{1}\right)}{t_{2}-t_{1}}<f\left(t_{1}, \psi\left(t_{1}\right)\right)+\frac{4}{2^{n}}+\varepsilon .
$$

Letting $n \rightarrow \infty$, we get

$$
f\left(t_{1}, \psi\left(t_{1}\right)\right)-\varepsilon \leqq \frac{\psi\left(t_{2}\right)-\psi\left(t_{1}\right)}{t_{2}-t_{1}} \leqq f\left(t_{1}, \psi\left(t_{1}\right)\right)+\varepsilon .
$$

This means that $\psi^{\prime}\left(t_{1}\right)=f\left(t_{1}, \psi\left(t_{1}\right)\right)$.

That $\psi$ is maximal follows from an application of the lemma, since $\phi_{n}^{\prime}$ $>f\left(t, \phi_{n}\right)$. Also, the lemma gives us that $w_{1} \leqq \psi \leqq w_{2}$.

The theorem is proved.

\section{Remarks}

REMARK 1. Since $f$ is continuous on a compact set $D$, it can be approximated uniformly by polynomials or by other functions that are continuous in the first variable and Lipschitz continuous in the second variable. In any case, the initial value problem $\phi^{\prime}=f_{n}(t, \phi)+4 / 2^{n}, \phi(0)=0$ can be solved uniquely on $J$. Thus there are functions $f_{n}$ that satisfy the hypothesis of Theorem $\mathbf{B}$.

Remark 2. One can also approximate $f$ by functions $f_{n}$ that are piecewise constant, but the corresponding solutions $\phi_{n}$ will only be piecewise continuously differentiable. However, one can ensure that the $f_{n}$ are defined in such a way that the $\phi_{n}$ satisfy $D_{+} \phi_{n}(t)=f_{n}\left(t, \phi_{n}\right)+4 / 2^{n}$ at corner points. This is enough for the proof of Theorem $B$ to go through.

REMARK 3. One can give a very short proof of Theorem B using uniform continuity of $f$ and Dini's theorem, while still avoiding the Ascoli lemma. The proof is the same except that we show $\psi$ is a solution by the following argument. Dini's theorem implies that $\phi_{n} \rightarrow \psi$ uniformly on $J$. (We are grateful to the referee for pointing out that uniform convergence also follows from the fact that if $f_{n} \rightarrow f$ pointwise on $J$ and the sequence $\left\{f_{n}\right\}$ is uniformly Lipschitz continuous, then $f$ is Lipschitz continuous and the convergence is uniform.) Thus $f\left(t, \phi_{n}(t)\right) \rightarrow f(t, \phi(t))$ by the uniform continuity of $f$. Also $F_{n}\left(t, \phi_{n}(t)\right)-f\left(t, \phi_{n}(t)\right) \rightarrow 0$ uniformly. Therefore,

$$
\phi(t)=\lim _{n} \phi_{n}(t)=\lim _{n} \int_{0}^{t} F_{n}\left(t, \phi_{n}(t)\right) d t
$$




$$
\begin{aligned}
& =\lim _{n} \int_{0}^{t}\left[F_{n}\left(t, \phi_{n}(t)\right)-f\left(t, \phi_{n}(t)\right)+f\left(t, \phi_{n}(t)\right)\right] d t \\
& =\int_{0}^{t} f(t, \phi(t)) d t .
\end{aligned}
$$

REMARK 4. The condition that the functions $\phi_{n}$ satisfy the equations $\phi_{n}^{\prime}=f_{n}\left(t, \phi_{n}\right)+4 / 2^{n}$ can be relaxed. It is sufficient that they satisfy the inequalities

$$
f_{n}\left(t, \phi_{n}\right)+\frac{4}{2^{n}}+\frac{1}{2^{n+1}} \geqq \phi_{n}^{\prime}(t) \geqq f_{n}\left(t, \phi_{n}\right)+\frac{4}{2^{n}} .
$$

\section{References}

[1] H. C. Kennedy, 'is there an elementary proof of Peano's existence theorem for first order differential equations?' Amer. Math. Monthly, 76 (1969), 1043-1045.

[2] G. Peano, Opere Scelte, vol. 1 (Edizioni Cremonese, 1957).

[3] O. Perron, 'Ein neuer Existenzbeweis für die Integrale der Differentialgleichung $y^{\prime}=f(x, y)$ ', Math. Ann. 76 (1915), 471-484.

[4] W. Walter, 'There is an elementary proof of Peano's existence theorem', Amer. Math. Monthly, 78 (1971), 170-173.

[5] H. Grunsky, 'Ein konstrucktiver Beweis für die Lösbarkeit der Differentialgleichung $y^{\prime}=$ $f(x, y)$ bei stetigem $f(x, y)$ ', Jahresber. Deutsch. Math. Ver., Abt. 1, 63 (1960), 78-84.

[6] W. Walter, Differential and Integral Inequalities (Springer Verlag, 1970).

[7] A. K. Aziz and J. B. Diaz, 'On Pompeiu's proof of the mean-value theorem of the differential calculus of real-valued functions', Contributions to Differential Equations, 1 (1963), 467-481.

[8] J. B. Diaz and R. Výborný, 'On mean value theorems for strongly continuous vector valued functions', Contributions to Differential Equations, 3 (1964), 107-118.

Department of Mathematics

University of Queensland

Australia. 